\title{
Spatial Control of Condensation on Chemically Homogeneous Pillar-Built Surfaces
}

\section{Mandsberg, Nikolaj Kofoed ; Taboryski, Rafael J.}

\section{Published in:}

Langmuir

Link to article, DOI:

10.1021/acs.langmuir.7b01159

Publication date:

2017

Document Version

Peer reviewed version

Link back to DTU Orbit

Citation (APA):

Mandsberg, N. K., \& Taboryski, R. J. (2017). Spatial Control of Condensation on Chemically Homogeneous Pillar-Built Surfaces. Langmuir, 33(21), 5197-5203. https://doi.org/10.1021/acs.langmuir.7b01159

\section{General rights}

Copyright and moral rights for the publications made accessible in the public portal are retained by the authors and/or other copyright owners and it is a condition of accessing publications that users recognise and abide by the legal requirements associated with these rights.

- Users may download and print one copy of any publication from the public portal for the purpose of private study or research.

- You may not further distribute the material or use it for any profit-making activity or commercial gain

- You may freely distribute the URL identifying the publication in the public portal

If you believe that this document breaches copyright please contact us providing details, and we will remove access to the work immediately and investigate your claim. 


\title{
Spatial Control of Condensation on Chemically Homogeneous Pillar-Built Surfaces
}

\author{
Nikolaj Kofoed Mandsberg and Rafael Taboryski* \\ Department of Micro- and Nanotechnology, Technical University of Denmark, 2800 Kongens Lyngby, \\ Denmark
}

\section{* rata@nanotech.dtu.dk}

Key words: Spatial control, chemically homogeneous, topography, focusing diffusive flux, dropwise condensation, nucleation point density, heterogeneous nucleation

\begin{abstract}
The random nature of dropwise condensation impedes spatial control hereof and its use for creating microdroplet arrays; yet, here we demonstrate spatial control of dropwise condensation on a chemically homogenous pillar array surface yielding $\sim 8000$ droplets $/ \mathrm{mm}^{2}$ under normal atmospheric pressure conditions. The studied pillar array surface is defined by photolithography and etched in silicon by deep reactive ion etching. Subsequently, the surface is covered with a self-assembled monolayer of perfluorodecyltrichlorosilane (FDTS) to render the surface hydrophobic. To obtain a perfect droplet array, with one droplet per pillar, we exploit a phenomenon, where the water vapor flux is focused toward the apices of surface asperities by diffusion, while matching the nucleation point density to the array dimensions. Matching is here achieved through variation of interpillar distance and vapor flow conditions.
\end{abstract}

\section{Introduction}

Much effort to understand the governing mechanisms of dropwise condensation has been done through several decades. ${ }^{1,2}$ A complete description is still missing due to the complexity of the phenomenon, and still today, researchers are giving much attention to this topic, owing to its importance for several scientific fields, such as: Energy conversion ${ }^{3,4}$, water harvesting ${ }^{5-7}$, thermal management systems ${ }^{8-12}$, and anti-icing ${ }^{13-}$ 16 .

In particular, an improved understanding will unlock the possibility of engineering the position of condensed droplets on a surface. The random nature of condensation in both time and space impedes spatial control of condensation. ${ }^{17}$ However, the ability to control the spatial positions of condensed 
droplets would allow the creation of microdroplet arrays, which could be a game changer for assays, e.g. biochemical assays ${ }^{18}$. For a surface, in general, two features can be changed: The chemistry ${ }^{19}$ and the topography ${ }^{20}$. A surface for spatial control of condensing droplets could then be imagined to originate from any of the three possible combinations. In 2009 Varanasi et al. ${ }^{21}$ showed how the combination of both chemistry and topography gives access to spatial control, and subsequently, others also exploited this chemistry-topography combination ${ }^{22}$. Quickly researchers also achieved spatial control using solely chemistry. Again it inspired many to follow this path. ${ }^{14,23,24}$ However, to the best of our knowledge, none has been able to cope with the pure-topography challenge, which is of particular interest as it allows for easy upscaling of fabrication using techniques such as nanoimprint lithography, injection molding, and rollto-roll extrusion coating. ${ }^{25-30}$ Attempts to obtain spatial control has been made; however, the level of control is still far from sufficient to create regular microdroplet arrays. ${ }^{31-33}$ In 2016 Park et al. made a study of "Condensation on slippery asymmetric bumps" and simultaneously made a critical observation. ${ }^{7}$ They saw how the apices of the bumps were subject to an increased condensation and predicted the existence of the "focusing diffusive flux" phenomenon.

Exercising the idea of focusing diffusive flux we here show how to achieve spatial control of condensation on chemically homogeneous surfaces. We condense water vapor on micropillars similar to the ones of many prior studies of which, however, none has convincingly reported the observation of spatial control. ${ }^{35}$ ${ }^{37}$ The explanation is that spatial control requires attention to surface structures, but equal attention is required to how the vapor is introduced. We find that an "airbrush" method where a precooled and dry surface is stroke by a vapor jet is the key to success. 


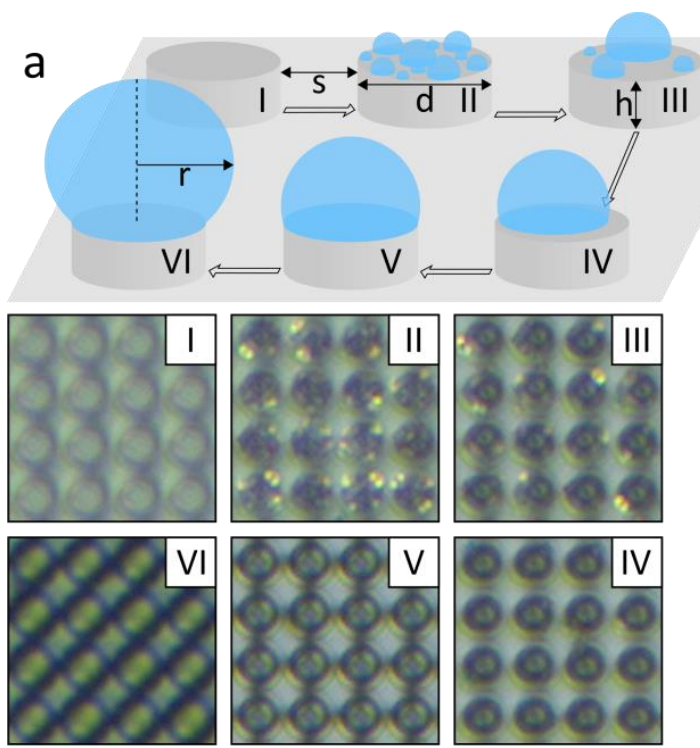

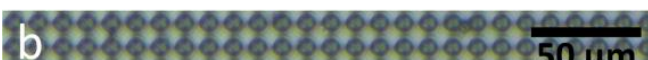

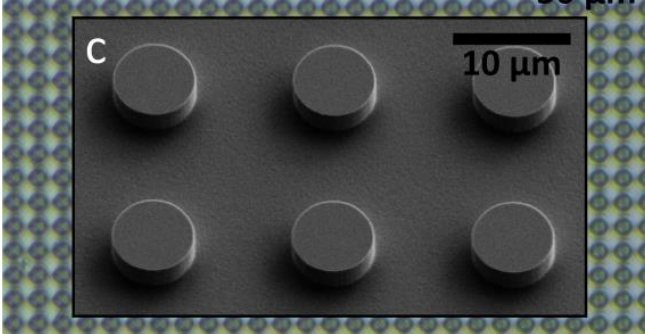

\%ั

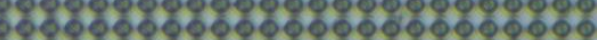

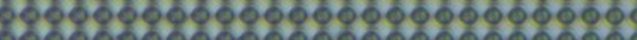

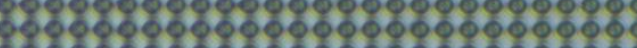

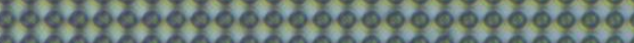

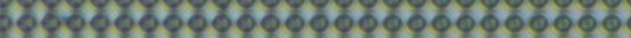

కరై

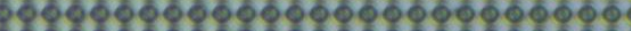

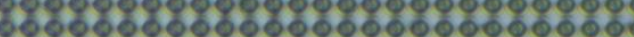

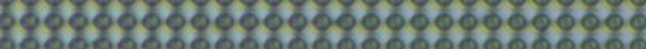

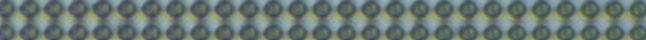

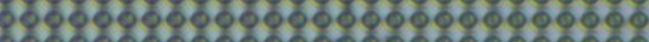

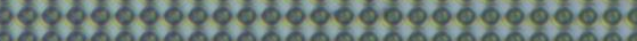

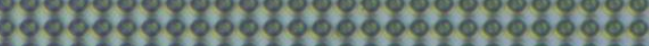

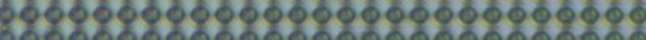

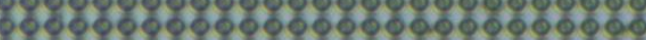

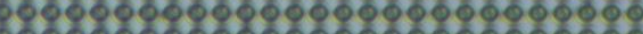

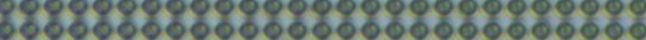

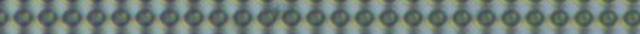

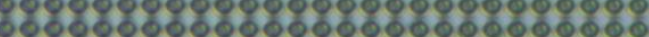

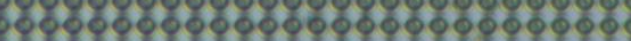

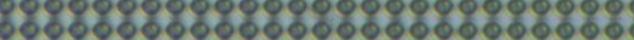

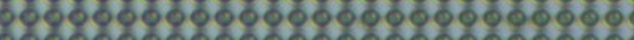

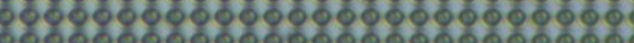

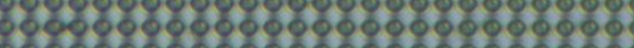

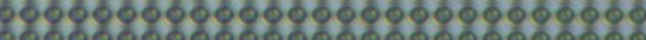

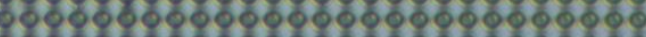

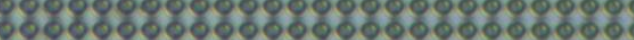

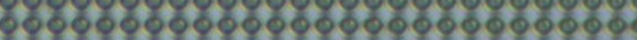

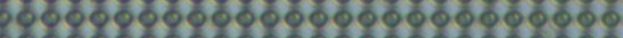

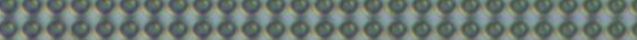

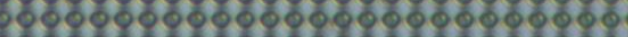

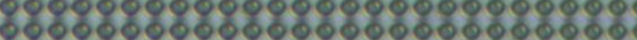

5060రం

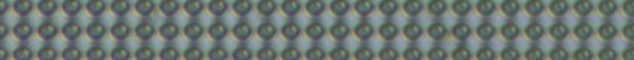


Figure 1: The genesis and growth of droplet arrays on circular pillars. The pillars have diameter $d$, a spacing of $s$, and a height of h. a) Schematic illustration and optical images of the genesis of the droplet array. The widths of the images are $44 \mu \mathrm{m}$. (I) Chilled, dry, chemically homogeneous pillar array. (II) Introduction of water vapor causes several droplets to nucleate on tops of the pillars. The bright droplets are a consequence of them being present at the very edge of the pillar causing an increased reflection due to index mismatching ${ }^{28}$. (III) Droplets fuses one-by-one causing the growth of one primary droplet. (IV) One droplet is now present on each pillar, but with a base radius smaller than the pillar radius. ( $V$ ) Continued growth of the individual droplet occurs until the triple-line coincides with the pillar edge. (VI) The droplet reaches a maximum apparent radius, $r$, determined by the array period. Further growth leads to coalescence of neighboring droplets and a breakdown of the regular droplet array. b) The optical image shows the demonstration of a large droplet array with one droplet per pillar. This particular array dimensioning allows for $\sim 8000$ droplets $/ \mathrm{mm}^{2}$. c) Scanning Electron Microscopy image of the pillar array.

In Figure 1a the mechanism of microdroplet array growth is illustrated and documented optically. Six phases are shown. (I) The micropillar topography, used in this study, is cooled below the dew point. (II) Vapor is introduced and many small droplets nucleate, but only on top of the pillar. (III) Continued condensation leads to coalescence and leaves one primary droplet. (IV) Only one droplet is present at each micropillar. (V) The droplet triple-line coincides with the edge of the pillar. (VI) A maximum apparent radius, $r=(s+d) / 2$, is reached and further condensation leads to interpillar droplet coalescence and loss of the array regularity. In Figure $\mathbf{1 b}$ it is demonstrated that an array of thousands of droplets is possible. In this particular case the array density is $\sim 8000$ droplets $/ \mathrm{mm}^{2}$. A Scanning Electron Microscopy image of the micro structure subject to the focusing diffusive flux is seen in Figure 1c.

\section{Experimental Section}

Sample fabrication: $100 \mathrm{~mm}<100>\mathrm{p}$-doped wafers were treated with hexamethyldisilazane (HMDS) to enhance adhesion, and subsequently spin coated (Süss MicroTec Gamma 2M spin coater) with $1.5 \mu \mathrm{m}$ positive tone photoresist (AZ Mir 701). The resist was soft baked at $90^{\circ} \mathrm{C}$ for 60 seconds to evaporate residual solvents. Afterward, the resist was exposed for 25 seconds with an intensity of $7.0 \mathrm{~mW} / \mathrm{cm}^{2}$ on a mask aligner (SÜSS MA6) in hard contact mode with the desired array pattern. The resist was then baked at $110^{\circ} \mathrm{C}$ for 60 seconds to maximize process latitudes and to mitigate issues with standing wave effects caused by monochromatic exposure, and thereafter, developed in AZ 726 MIF (Metal lon Free) for 60 seconds (Süss MicroTec Gamma 2M developer). The pillar array was transferred into silicon using reactive ion etch (RIE, Pegasus D-RIE, STS, UK) in a Bosch process for $c$ cycles to achieve different pillar heights, $h$. 
The resist was stripped by plasma ashing in a mixture of $400 \mathrm{sccm} \mathrm{O} \mathrm{O}_{2}$ and $70 \mathrm{sccm} \mathrm{N2}$ at $1000 \mathrm{~W}$ for 25 minutes. Finally, deposition of a self-assembled hydrophobic monolayer using the precursor perfluorodecyltrichlorosilane (FDTS) in molecular vapor deposition (MVD, MVD 100, MST, USA) to render the surface hydrophobic.

Condensation procedure: The experimental setup is sketched in Figure 2a. A $\sim 3 \times 3 \mathrm{~mm}^{2}$ micropillar substrate was fastened in thermal paste on top of a Peltier cooled copper plate (Figure 2 b). (8.0 00.5$) \mathrm{L} / \mathrm{min}$ of nitrogen flow was introduced in the vicinity to deplete water vapor from the substrate. The copper plate was cooled to $(5.0 \pm 0.3){ }^{\circ} \mathrm{C}$ and left for $>1$ minute allowing temperature stabilization of the silicon chip. Nitrogen was saturated with water by bubbling it through de-ionized (DI) water with a temperature of $(21 \pm 1)^{\circ} \mathrm{C}$ and led through another saturated atmosphere of DI water vapor (see Figure 2a). The nitrogen line was closed; the saturated vapor line opened with flowrate $(0.5 \pm 0.05) \mathrm{L} / \mathrm{min}$; and the tube was translated at $|\vec{u}|=(16.3 \pm 0.7) \mathrm{mm} / \mathrm{s}$ along the edge of the sample in an "airbrush" action as indicated in Figure 2c. Photographs of the setup to realize the translation are found in the Supplementary Information Figure $\mathbf{S 1}$ and $\mathbf{S 2}$, and the motion was caused using fishing line hauled by a LEGO MINDSTORMS Servo Motor programmed in LEGO MINDSTORMS EV3 Home Edition software. The translation speed was determined by video recordings (PLAYSTATION Eye camera), and analysis hereof in the open source software Tracker 4.9x (see SI, Figure S3 for displacement graphs). The distance from the outlet to sample

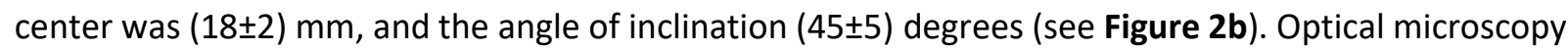
images (ZEISS Axio Zoom.V16, ZEISS, Germany) were recorded every second for 50 seconds during condensation. 

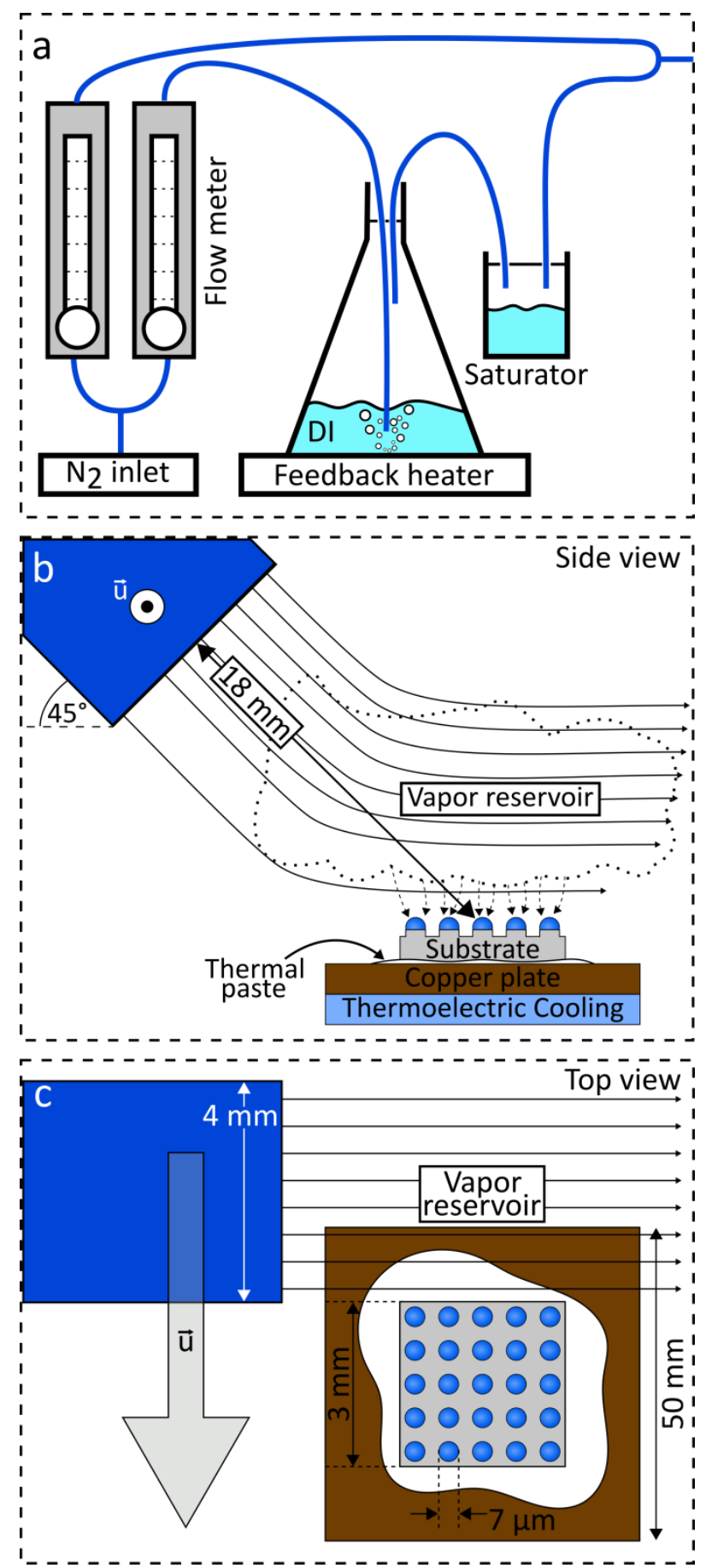

Figure 2: Overview of the experimental setup for the "airbrush" method. a) Two lines (blue tubes) of nitrogen flow are controlled by mechanical flowmeters. One line is used to deplete water vapor from the chip surface. The other line is bubbled through DI water, whose temperature is held constant using a feedback heater, and afterward passed through a saturator. b) Side view. The saturated vapor flows over the substrate, thereby creating a vapor reservoir for diffusion toward the substrate. c) Top view. The vapor is introduced by sideward movement of the vapor tube, thereby making a sudden appearance of the vapor reservoir. 


\section{Results and Discussion}

Pillar height variation: Condensation experiments were performed on chemically homogeneous substrates with micropillar arrays. Prior to substrate cooling, below the dew point, its vicinity was depleted for water molecules by flushing with dry nitrogen. Substrate cooling to $5{ }^{\circ} \mathrm{C}$ and subsequent introduction of a conditioned, saturated water vapor reservoir led to condensation. In Figure 3-left a 1 dimensional representative of the log intensity spectrum obtained from a Fourier transform (see SI for details on the Fourier transform) is shown. The spectra are generated from the optical recordings of condensation experiments (Figure 3-right) performed on pillar arrays differing only in their height, $h$. The pillar diameter, $d$, and spacing, $s$, were fixed to 7 and $4 \mu \mathrm{m}$, respectively. Pillar heights of $0,0.37,1.1$, and $2.6 \mu \mathrm{m}$ were tested, and the result is a transition into a perfectly periodic, defect-free microdroplet array when the height is increased. The $h=0 \mu \mathrm{m}$ signal is very noisy as a consequence of the random nature of condensation, but already at $h=0.37 \mu \mathrm{m}$ peaks emerge. The fundamental peak is located at $\sim 11 \mu \mathrm{m}$ in agreement with the structure period, $d+s$. However, for the height of $0.37 \mu \mathrm{m}$ the spatial control is not obvious, even though the Fourier transform provides evidence of a structure-condensation interaction. Increasing the height to $1.1 \mu \mathrm{m}$ the spatial control becomes very pronounced as observed in the optical image, and for $2.6 \mu \mathrm{m}$ all array defects have vanished. It is evident that the pillar height is a crucial parameter. 


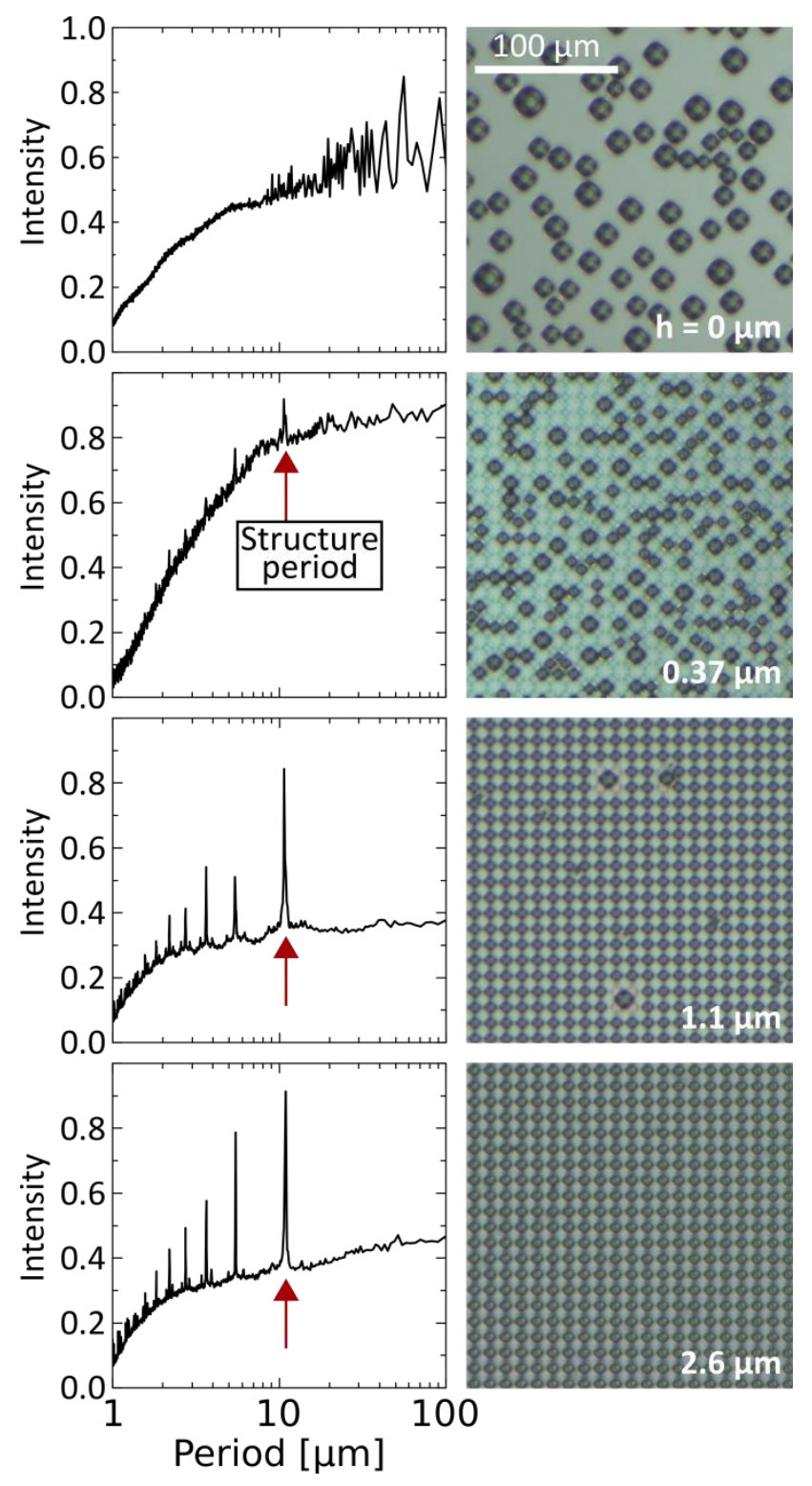

Figure 3: Similar condensation experiments performed on pillar arrays differing only in their height, $h$. (Left) A 1-dimensional representation of the intensity spectrum obtained from a Fourier transform of the optical photograph on the (right). A clear transition to a periodic microdroplet array is observed as the pillar height increases; seen by a fundamental intensity peak emerging at the $11 \mu \mathrm{m}$ period of the pillar array.

Pillar spacing variation: Our protocol with initial vapor depletion, then cooling, and lastly an introduction of a vapor reservoir seems straightforward. It may raise the question why spatial control on similar micropillars was not observed until now. We believe it all boils down to a required match between the array dimensions and the nucleation point density. Figure 4 shows the importance of this matching, where similar condensation experiments were performed on pillar arrays differing only in their spacing, s. In Figure 4-left an early stage, $t_{1}$, in the droplet growth is shown, and Figure 4-right is 24 seconds later, $t_{2}$. For 
$s=2 \mu \mathrm{m}$ the diffuse image turns into a regular array of droplets. The same applies for $4 \mu \mathrm{m}$ despite the existence of initial (bright) edge droplets, which were also shown in Figure 1a(II-III). An increase to $8 \mu \mathrm{m}$ allows for interstitial droplets, and continued condensation leads to interpillar coalescence and chaos at $t_{2}$. The nucleation point density is, among other, dependent on temperature, vapor humidity, and flow conditions, ${ }^{38,39}$ why the introduction of the vapor is critical to adjust. In Supporting Information Figure S4 we show how the variation of the flow rate influences the quality of the microdroplet array. Additionally, when using dry etching for silicon fabrication, one is certain to introduce some degree of nanoroughness. With nucleation radii of $\sim 2 \mathrm{~nm}^{21}$ the nanoscale topography needs to be taken into account, ${ }^{40,41}$. However, it is our impression that presently the level of understanding still does not allow precise engineering of nucleation defects.

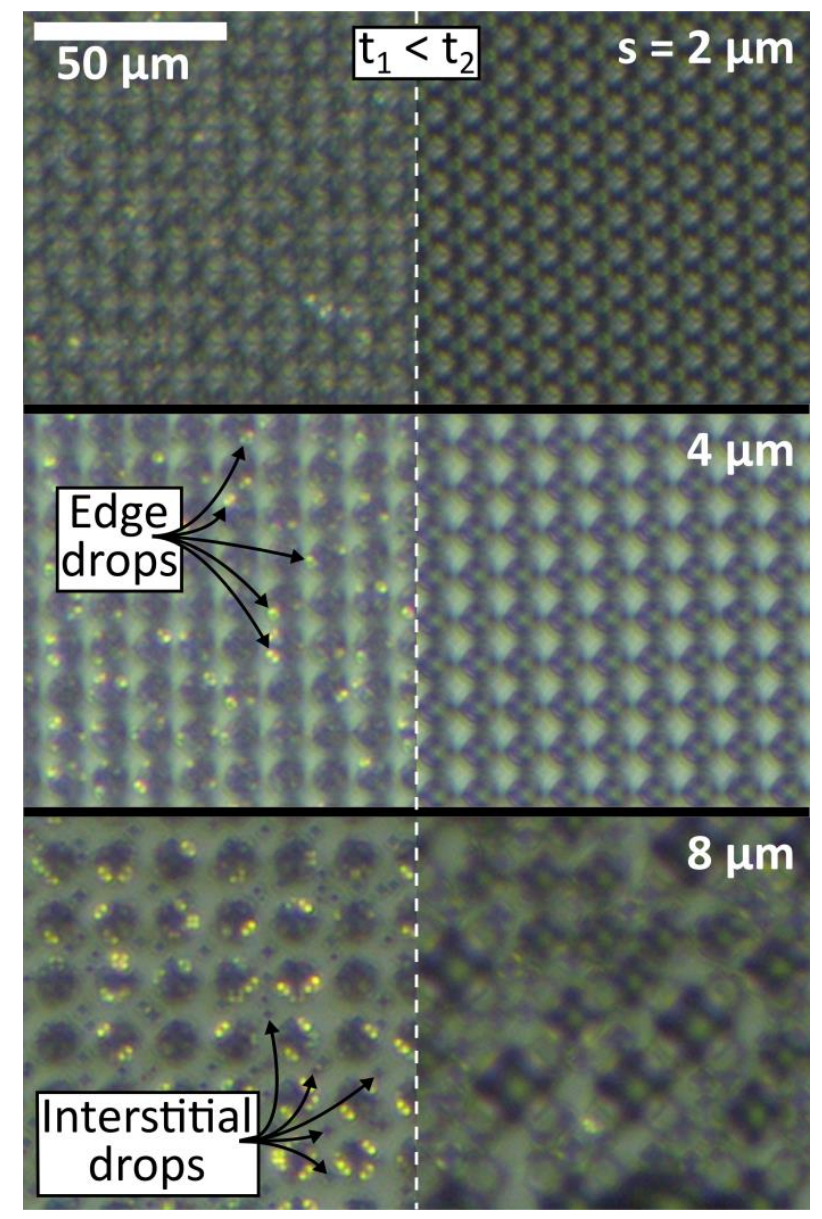

Figure 4: Similar condensation experiments performed on pillar arrays differing only in their spacing, $s$, as defined in Figure $1 a$. On the $s=2$ and $4 \mu \mathrm{m}$ case the nucleation density matches the pillar surface coverage. For $4 \mu \mathrm{m}$ many edge droplets (bright droplets) are present at $t_{1}$, but during continued condensation these coalesce within the single pillar. It results in a microdroplet array at $t_{2}$ of similar high 
quality to that of $2 \mu \mathrm{m}$. For $s=8 \mu \mathrm{m}$ the pillar spacing is too large allowing for interstitial drops (small dark droplets), that with continued condensation leads to interpillar coalescence and chaos at $t_{2}$.

Finite element modeling: Using the finite element method, qualitative numerical simulations were carried out in order to explain the preferred location of condensation. Figure 5a shows an initial simulation of the flow conditions in the experimental setup. From the no slip-boundary conditions a boundary layer of some thickness, $H$, arises. This corroborates the idea of having a constant value reservoir in the diffusion problem being responsible for supplying vapor to initiate droplet condensation. Based on the reservoir idea, the simulation domain seen in Figure $\mathbf{5 b}$ was set up and solved. Constraining the material surface concentration to zero simulates the adsorption of water vapor molecules, while a constraint to unity simulates the constant water vapor source (see SI for details on the simulation). In Figure $\mathbf{5 c}$ the timedependent solution to the diffusion equation, $D \nabla^{2} c=\partial_{t} c$, where $D$ is diffusion coefficient, and $c$ is the normalized dimensionless concentration of water molecules, is shown with the spatially averaged flux toward the pillar top and the interpillar region. The observation that the flux toward the pillar top at any time is larger than between pillars is equivalent to an enhanced probability of having nucleation on top of the pillars. Initially the flux is zero, which is a consequence of an initial bulk concentration of 0 . In contrary, when first introducing vapor (bulk value $c=1$ ), and hereafter cooling the sample (sample surface value $c=0$ ) would result in a flux decreasing with time, thereby making initial nucleation in the interpillar region much more likely. This is part of the explanation why the use of environmental electron scanning microscopes has not revealed spatial control on chemically homogenous surfaces. Figure $\mathbf{5} \mathbf{d}$ shows the stationary solution to the diffusion problem for three different pillar aspect ratios (height:diameter). The larger flux contrast on the higher pillars indicates a screening of the interpillar regions, which favors condensation on the pillar tops. This is exactly the "focusing diffusive flux" phenomenon, and it causes the initial nucleation to be on the pillar tops. Similar focusing effects were reported by several groups, and lately by Sun and Rykaczewski, where micro-patches of hygroscopic liquid were used as sinks for water vapor, while the surrounding area was rendered water repellent by a hydrophobic surface chemistry. ${ }^{42,43}$ In a FEM simulation Sun and Rykaczewski employed constant value boundary conditions at the surface of the hygroscopic liquid and a zero flux condition on the hydrophobic areas to explain the observed humidity sink effect. In Figure 5e, we present a simulation without the assumption of zero flux and find how an enhancement of the selectivity is present even for a conservative scenario without the hygroscopic effect. Figure $\mathbf{5 e}$ solves the stationary diffusion problem (from Figure $\mathbf{5 d}$ ) but has been altered to include also a hemispherical droplet to investigate how the selectivity evolves. Figure $\mathbf{5 e - l e f t ~ s i m u l a t e s ~ t h e ~ c a s e ~ b e f o r e ~}$ any water is present on the pillar top. The flux toward pillar top to interpillar region ratio is 5 . In Figure 5eright includes the condensate and consequently the flux ratio increases to 13 . This $165 \%$ increase shows 
how the flux toward the pillar tops is a self-enhancing effect even under a conservative, pure geometry consideration. The effect causes further impedance of interpillar droplet nucleation at advanced times and makes this technique for droplet array creation more robust.
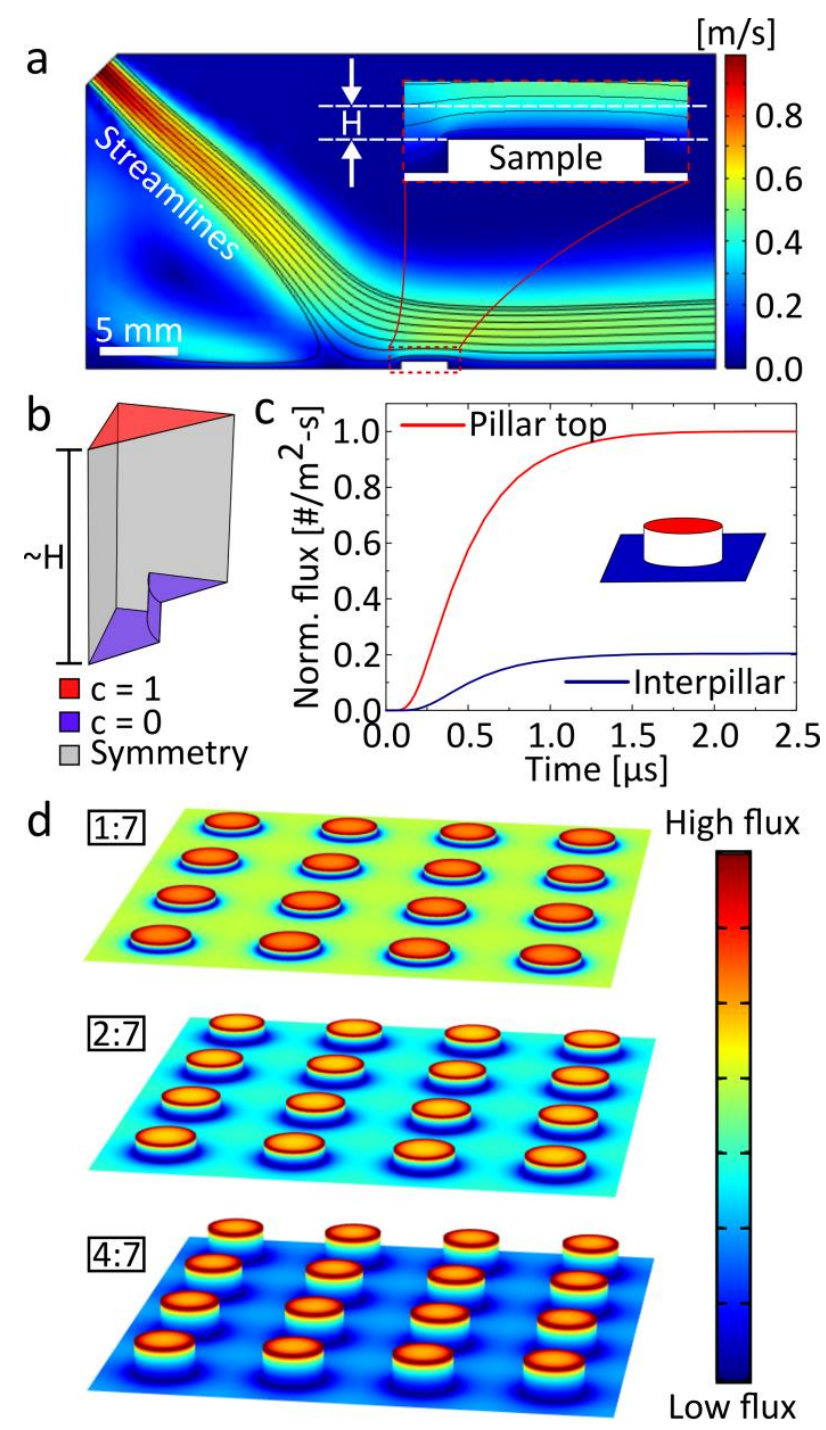

e

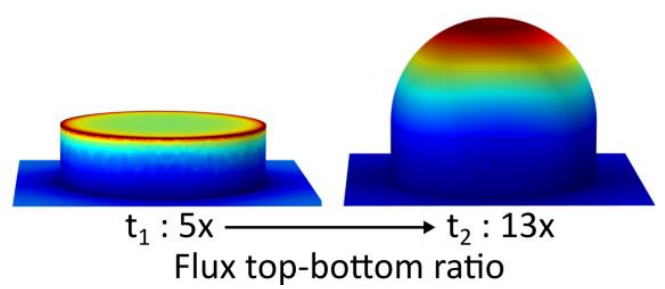

Figure 5: Finite element simulations for a qualitative explanation of "focusing diffusive flux". a) Simulation of the flow toward the sample illustrates the boundary layer of some height, $H$. Above this height a rapid resupply of vapor emulates a constant value reservoir to be used as a boundary condition in a diffusion problem. b) Boundary conditions for the diffusion problem. As suggested the concentration is set constant, 
$c=1$, away from the surface. Under conditions causing condensation, an infinitely fast consumptions of vapor molecules at the surface is assumed, $c=0$. Symmetry boundary conditions have been imposed to simulate an infinite array of micro-pillars. c) Time-dependent solution to the diffusion problem with an initial bulk concentration of 0 . It is observed that at any point in time, the flux toward the pillar top is larger than in the interpillar region. d) Stationary solution to the diffusion problem for different pillar heights. The larger flux contrast for the higher pillars indicates a screening of the interpillar region, which favors condensation on the pillar tops. e) Stationary solution to the diffusion problem at two different droplet growth stages. $t_{1}$ is before any condense on the pillar tops. $t_{2}$ emulates the geometrical alternation caused by a condensed hemispherical droplet and the consequences for the flux. A significant increase in the selectivity toward the pillar tops is evident.

To match the nucleation point density to the pillar height and interpillar distance a finely tuned flow is required. However, once the surface is prepped (array has occurred once) then it is much easier to get the array perfect. The investigated surface was engineered with only one level of texture, i.e. no hierarchy, and still it was able to focus the diffusive flux. Xu et al. conducted an analytical study of the heterogeneous nucleation for conical structures. ${ }^{44}$ They found how conical cavities have a lower nucleation free energy barrier compared to apices and planar substrates. We thus believe that the nucleation site density could be locally enhanced and the selectivity between pillars and the surrounding area could be further increased by introduction of micro cavities, or suitable nanotexturing, ${ }^{11,33,45}$ on the pillar tops.

Leach et al. found that droplets smaller than $50 \mu \mathrm{m}$ in diameter grow by diffusion of water adsorbed on the substrate to the droplet perimeter, ${ }^{9}$ which may also be the case for our pillar substrate. However here, when this type migration is restricted to take place on top of the pillars, it results in droplet coalescence as shown in Figure 1a. To achieve an even larger array density requires fabrication methods to form arrays with pillars of nanometric size $\mathrm{e}^{25,46,47}$ and following a necessity to match the nucleation point density, which Castillo et al. found to be tunable through the relative humidity, ${ }^{38}$. In addition, Castillo et al. found how the droplet growth rate increases with the relative humidity allowing for faster array growth, while Varanasi predicted ${ }^{21}$ that if this spatial control was possible, droplets could be made to preferentially nucleate on post tops, thereby forcing Cassie- type behavior on textured surfaces even under condensation. See also Zu et al. ${ }^{34}$. Our observation, however, does not seem to support this prediction as the droplets collapse in between pillars upon coalescence (see SI, Figure S5).

\section{Summary and Conclusion:}


To summarize, we fabricated and studied condensation of water vapor on a chemically homogenous hydrophobic pillar array surface. In conclusion, we find that the creation of a regular microdroplet array is possible by condensation using pure topography, and can be achieved only when the nucleation point density is matched to the pillar height and interpillar distance, through the way the vapor is introduced. We find that an "airbrush" action, where a precooled and dry surface is stroked by a vapor jet is a key to success. Hence, two important aspects need to be considered; both the topography and the way the vapor is introduced are critically important.

\section{Supporting Information}

Experimental setup, Flow translation speed, Flow speed variation, Fourier spectrum, Finite Element Method, Transition into Wenzel state upon inter-pillar coalescence

\section{References}

1. Enright, R.; Miljkovic, N.; Alvarado, J. L.; Kim, K.; Rose, J. W., DROPWISE CONDENSATION ON MICRO- AND NANOSTRUCTURED SURFACES. Nanoscale and Microscale Thermophysical Engineering 2014, 18 (3), 223-250.

2. Rose, J. W., Dropwise condensation theory and experiment: a review. Proceedings of the Institution of Mechanical Engineers Part a-Journal of Power and Energy 2002, 216 (A2), 115-128.

3. Beer, J. M., High efficiency electric power generation: The environmental role. Progress in Energy and Combustion Science 2007, 33 (2), 107-134.

4. Glicksman, L. R.; Hunt, A. W., NUMERICAL SIMULATION OF DROPWISE CONDENSATION. International Journal of Heat and Mass Transfer 1972, 15 (11), 2251-+.

5. $\quad$ Andrews, H. G.; Eccles, E. A.; Schofield, W. C. E.; Badyal, J. P. S., Three-Dimensional Hierarchical Structures for Fog Harvesting. Langmuir 2011, 27 (7), 3798-3802.

6. Bai, H.; Wang, L.; Ju, J.; Sun, R. Z.; Zheng, Y. M.; Jiang, L., Efficient Water Collection on Integrative Bioinspired Surfaces with Star-Shaped Wettability Patterns. Advanced Materials 2014, 26 (29), 5025-5030.

7. Park, K. C.; Kim, P.; Grinthal, A.; He, N.; Fox, D.; Weaver, J. C.; Aizenberg, J., Condensation on slippery asymmetric bumps. Nature 2016, 531 (7592), 78-82.

8. Miljkovic, N.; Wang, E. N., Modeling and optimization of hybrid solar thermoelectric systems with thermosyphons. Solar Energy 2011, 85 (11), 2843-2855.

9. Leach, R. N.; Stevens, F.; Langford, S. C.; Dickinson, J. T., Dropwise condensation:

Experiments and simulations of nucleation and growth of water drops in a cooling system. Langmuir 2006, 22 (21), 8864-8872.

10. Rykaczewski, K.; Scott, J. H. J.; Rajauria, S.; Chinn, J.; Chinn, A. M.; Jones, W., Three dimensional aspects of droplet coalescence during dropwise condensation on superhydrophobic surfaces. Soft Matter 2011, 7 (19), 8749-8752.

11. Chen, X. M.; Wu, J.; Ma, R. Y.; Hua, M.; Koratkar, N.; Yao, S. H.; Wang, Z. K., Nanograssed Micropyramidal Architectures for Continuous Dropwise Condensation. Advanced Functional Materials 2011, 21 (24), 4617-4623.

12. Xiao, R.; Miljkovic, N.; Enright, R.; Wang, E. N., Immersion Condensation on Oil-Infused Heterogeneous Surfaces for Enhanced Heat Transfer. Scientific Reports 2013, 3. 
14. Boreyko, J. B.; Hansen, R. R.; Murphy, K. R.; Nath, S.; Retterer, S. T.; Collier, C. P., Controlling condensation and frost growth with chemical micropatterns. Scientific Reports 2016, 6.

15. Mishchenko, L.; Khan, M.; Aizenberg, J.; Hatton, B. D., Spatial Control of Condensation and Freezing on Superhydrophobic Surfaces with Hydrophilic Patches. Advanced Functional Materials 2013, 23 (36), 4577-4584.

16. Chen, X. M.; Ma, R. Y.; Zhou, H. B.; Zhou, X. F.; Che, L. F.; Yao, S. H.; Wang, Z. K., Activating the Microscale Edge Effect in a Hierarchical Surface for Frosting Suppression and Defrosting Promotion. Scientific Reports 2013, 3.

17. Nath, S.; Boreyko, J. B., On Localized Vapor Pressure Gradients Governing Condensation and Frost Phenomena. Langmuir 2016, 32 (33), 8350-8365.

18. Hu, S. W.; Xu, B. Y.; Ye, W. K.; Xia, X. H.; Chen, H. Y.; Xu, J. J., Versatile Microfluidic Droplets Array for Bioanalysis. Acs Applied Materials \& Interfaces 2015, 7 (1), 935-940.

19. Larsen, S. T.; Taboryski, R., A Cassie-Like Law Using Triple Phase Boundary Line Fractions for Faceted Droplets on Chemically Heterogeneous Surfaces. Langmuir 2009, 25 (3), 1282-1284.

20. Nikolaj Kofoed Mandsberg and Rafael, T., The rose petal effect and the role of advancing water contact angles for drop confinement. Surface Topography: Metrology and Properties 2017, 5 (2), 024001.

21. Varanasi, K. K.; Hsu, M.; Bhate, N.; Yang, W. S.; Deng, T., Spatial control in the heterogeneous nucleation of water. Applied Physics Letters 2009, 95 (9).

22. Hou, Y. M.; Yu, M.; Chen, X. M.; Wang, Z. K.; Yao, S. H., Recurrent Filmwise and Dropwise Condensation on a Beetle Mimetic Surface. Acs Nano 2015, 9 (1), 71-81.

23. Yamada, Y.; Ikuta, T.; Nishiyama, T.; Takahashi, K.; Takata, Y., Droplet Nucleation on a WellDefined Hydrophilic-Hydrophobic Surface of 10 nm Order Resolution. Langmuir 2014, 30 (48), 1453214537.

24. Ghosh, A.; Beaini, S.; Zhang, B. J.; Ganguly, R.; Megaridis, C. M., Enhancing Dropwise Condensation through Bioinspired Wettability Patterning. Langmuir 2014, 30 (43), 13103-13115.

25. Murthy, S.; Matschuk, M.; Huang, Q.; Mandsberg, N. K.; Feidenhans'I, N. A.; Johansen, P.; Christensen, L.; Pranov, H.; Kofod, G.; Pedersen, H. C.; Hassager, O.; Taboryski, R., Fabrication of Nanostructures by Roll-to-Roll Extrusion Coating. Advanced Engineering Materials 2016, 18 (4), 484-489. 26. Ahn, S. H.; Guo, L. J., High-speed roll-to-roll nanoimprint lithography on flexible plastic substrates. Advanced Materials 2008, 20 (11), 2044-+.

27. Tanzi, S.; Ostergaard, P. F.; Matteucci, M.; Christiansen, T. L.; Cech, J.; Marie, R.; Taboryski, R., Fabrication of combined-scale nano- and microfluidic polymer systems using a multilevel dry etching, electroplating and molding process. Journal of Micromechanics and Microengineering 2012, 22 (11). 28. Sogaard, E.; Andersen, N. K.; Smistrup, K.; Larsen, S. T.; Sun, L.; Taboryski, R., Study of Transitions between Wetting States on Microcavity Arrays by Optical Transmission Microscopy. Langmuir 2014, 30 (43), 12960-12968.

29. Heyderman, L. J.; Schift, H.; David, C.; Gobrecht, J.; Schweizer, T., Flow behaviour of thin polymer films used for hot embossing lithography. Microelectronic Engineering 2000, 54 (3-4), 229-245. 30. Schift, H.; David, C.; Gabriel, M.; Gobrecht, J.; Heyderman, L. J.; Kaiser, W.; Koppel, S.; Scandella, L., Nanoreplication in polymers using hot embossing and injection molding. Microelectronic Engineering 2000, 53 (1-4), 171-174.

31. Medici, M. G.; Mongruel, A.; Royon, L.; Beysens, D., Edge effects on water droplet condensation. Physical Review E 2014, 90 (6).

32. Lo, C. W.; Wang, C. C.; Lu, M. C., Spatial Control of Heterogeneous Nucleation on the Superhydrophobic Nanowire Array. Advanced Functional Materials 2014, 24 (9), 1211-1217.

33. Cho, Y.; Shim, T. S.; Yang, S., Spatially Selective Nucleation and Growth of Water Droplets on Hierarchically Patterned Polymer Surfaces. Advanced Materials 2016, 28 (7), 1433-1439. 
34. Zu, Y. Q.; Yan, Y. Y., Single Droplet on Micro Square-Post Patterned Surfaces - Theoretical Model and Numerical Simulation. Scientific Reports 2016, 6.

$35 . \quad$ Narhe, R. D.; Beysens, D. A., Growth dynamics of water drops on a square-pattern rough hydrophobic surface. Langmuir 2007, 23 (12), 6486-6489.

36. Wier, K. A.; McCarthy, T. J., Condensation on ultrahydrophobic surfaces and its effect on droplet mobility: Ultrahydrophobic surfaces are not always water repellant. Langmuir 2006, 22 (6), 24332436.

37. Chen, C. H.; Cai, Q. J.; Tsai, C. L.; Chen, C. L.; Xiong, G. Y.; Yu, Y.; Ren, Z. F., Dropwise condensation on superhydrophobic surfaces with two-tier roughness. Applied Physics Letters 2007, 90 (17). 38. Castillo, J. E.; Weibel, J. A.; Garimella, S. V., The effect of relative humidity on dropwise condensation dynamics. International Journal of Heat and Mass Transfer 2015, 80, 759-766.

39. Zhou, K.; Chan, T. L., Analytical Approximation Schemes for Mean Nucleation Rate in Turbulent Flows. Aerosol Science and Technology 2014, 48 (5), 459-466.

40. Stoyanov, S., NUCLEATION ON POINT-DEFECTS. Journal of Crystal Growth 1974, 24 (OCT), 293-297.

41. Stenzel, H.; Bethge, H., INVESTIGATION OF HETEROGENEOUS NUCLEATION ON POINTDEFECTS. Thin Solid Films 1976, 32 (2), 267-270.

42. Sun, X. D.; Rykaczewski, K., Suppression of Frost Nucleation Achieved Using the Nanoengineered Integral Humidity Sink Effect. Acs Nano 2017, 11 (1), 906-917.

43. Sun, X. D.; Damle, V. G.; Uppal, A.; Linder, R.; Chandrashekar, S.; Mohan, A. R.; Rykaczewski, K., Inhibition of Condensation Frosting by Arrays of Hygroscopic Antifreeze Drops. Langmuir 2015, 31 (51), 13743-13752.

44. Xu, W.; Lan, Z.; Peng, B. L.; Wen, R. F.; Ma, X. H., Heterogeneous nucleation capability of conical microstructures for water droplets. Rsc Advances 2015, 5 (2), 812-818.

45. Schneider, L.; Laustsen, M.; Mandsberg, N.; Taboryski, R., The Influence of Structure Heights and Opening Angles of Micro- and Nanocones on the Macroscopic Surface Wetting Properties. Scientific Reports 2016, 6.

46. Jiang, B. Y.; Zhou, M. Y.; Weng, C.; Zhang, L.; Lv, H., Fabrication of nanopillar arrays by combining electroforming and injection molding. International Journal of Advanced Manufacturing Technology 2016, 86 (5-8), 1319-1328.

47. Kuo, C. W.; Shiu, J. Y.; Chen, P. L., Size- and shape-controlled fabrication of large-area periodic nanopillar arrays. Chemistry of Materials 2003, 15 (15), 2917-2920. 
TOC Figure

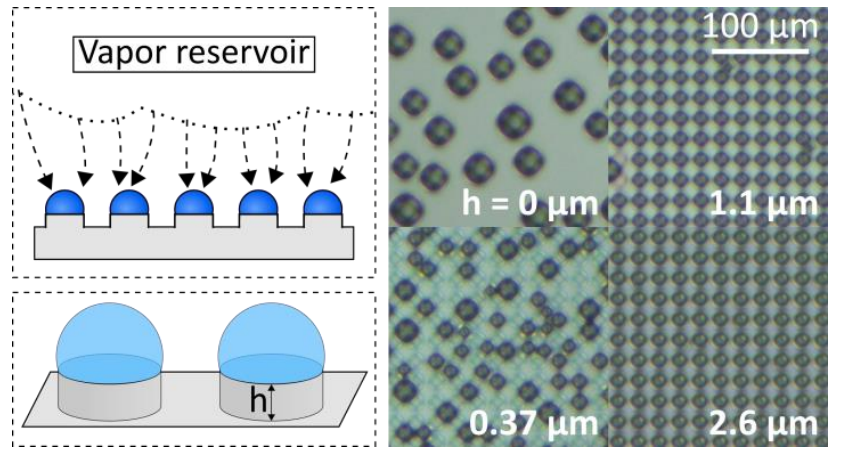

